J. Lake Sci. (湖泊科学) , 2006, 18(6):585-589

http:// www. jlakes. org. E-mail: jlakes@ niglas. ac.cn

(c) 2006 by Journal of Lake Sciences

\title{
太湖水华蓝藻底泥中复苏和水柱中生长的比较”
}

\author{
曹焕生 ${ }^{1,2}$, 孔繁翔 ${ }^{1 * *}$, 谭 啸 ${ }^{3}$,杨 洲 $^{3}$, 张 民 ${ }^{1,2}$, 邢 鹏 ${ }^{1,2}$ \\ (1: 中国科学院南京地理与湖泊研究所, 南京 210008) \\ (2:中国科学院研究生院, 北京 100049) \\ (3: 南京师范大学生命科学学院, 南京 210097)
}

\begin{abstract}
摘 要: 为了研究太湖底泥中蓝藻的复苏和水柱中的生长, 作者自行设计了原位藻类复苏收集器和生长培养器并安放在太湖梅梁 湾监测蓝藻复苏释放和生长. 实验在藻类的复苏期 (2005 年 3-6月)进行. 结果表明在复苏期太湖蓝藻的复苏呈现波动性增加, 4 月达到最大量. 水柱中蓝藻同步的比生长率和生长量也呈现波动性上升, 并在 5 月初达到最大值. 浮游动物的存在并没有对蓝藻 的生长造成显著的影响. 对底泥蓝藻释放和水柱生长进行比较, 底泥释放的蓝藻只占藻类生长量的很小一部分 $(<2.5 \%)$. 所以, 水柱中蓝藻的生长对其优势的确立和水华的形成具有重要的作用,今后的研究将主要集中在水柱中蓝藻的动态变化.
\end{abstract}

关键词:蓝藻;藻蓝素;复苏;生长率;太湖

\section{Comparison of recruitment from sediments with pelagic growth of cyanobacteria in Lake Taihu, China}

CAO Huansheng ${ }^{1,2}$, KONG Fanxiang ${ }^{1 *}$, TAN Xiao $^{3}$, YANG Zhou ${ }^{3}$, ZHANG Min ${ }^{1,2}$ \& XING Peng ${ }^{1,2}$

(1:Nanjing Institute of Geography and Limnology, Chinese Academy of Sciences, Nanjing 210008,P. R. China)

(2: Graduate School of Chinese Academy of Sciences, Beijing 100039, P. R. China)

(3:School of Life Science, Nanjing Normal University, Nanjing 210097, P. R. China)

Abstract: Cyanobacteria recruitment from sediments is an important seed bank for their pelagic abundance. We estimated cyanobacteria recruitment from sediments with migration traps in Lake Taihu from March to June 2005, measured in situ growth rate of cyanobacteria in the water column in specially designed chambers, and compared the sizes of both processes. Meanwhile the effect of zooplankton grazing on cyanobacteria growth rate was also measured. Cyanobacteria recruitment increased with some degree of fluctuation from March to June, peaking in April; in situ growth rate of cyanobacteria also showed a general increase at the same time, only reached maximum in early May. No obvious effect of zooplankton grazing was observed on the in situ cyanobacteria growth rate. Cyanobacteria recruitment only accounted for a small portion of the pelagic growth $(<2.5 \%)$ thereby suggesting that future research on cyanobacteria bloom development be focused on the pelagic populations in water column.

Keywords: Cyanobacteria; phycocyanin; recruitment; growth rate; Lake Taihu

近几十年来富营养湖泊中形成的蓝藻水华导致了的生态系统结构和功能失调, 造成了严重的社会经济损失, 成为亟待解决的环境问题 ${ }^{[1,2]}$. 蓝藻水华分为藻类的下沉和越冬 ${ }^{[3-7]}$ 、在底泥表面的复苏 ${ }^{[8-11]}$ 、水柱中生物量积累 和优势确立、上浮并形成水华等几个相互独立而又彼此相关的阶段 ${ }^{[12]}$. 其中蓝藻复苏是水华形成的关键过程, 即 在底泥表面越冬的蓝藻在环境条件适宜时恢复活性、开始生长并向水柱迁移的过程.复苏为水柱中蓝藻的生长提 供种源, 是水柱中蓝藻生物量积累的前提. 蓝藻复苏的研究在国内外已成为水华机理研究的热点 ${ }^{[8-11]}$. 但是多数

* 国家自然科学基金项目(40471045), 国家重点基础研究发展计划资助项目(2002CB412305), 中国科学院百人计划和南京地 理与湖泊研究所所长基金联合资助. 2005-11-22 收稿;2006-02-17 收修改稿. 曹焕生,男,1978 年生,博士研究生; Email: hscao@ niglas.ac.cn.

** 通讯联系人; E-mail: fxkong@ niglas. ac. cn. 
研究集中在深水湖泊,深水湖泊中蓝藻的复苏量只占蓝藻最大生物量的 $5 \%$ 左右 ${ }^{[9]}$. 即使如此,如果不考虑蓝藻的 复苏, 水华形成时蓝藻的生物量会减少 $50 \%{ }^{[13]}$. 浅水湖泊中的蓝藻复苏研究较少. Cao 等人对太湖中蓝藻的复苏 做了定量的研究后认为, 底泥中复苏释放的蓝藻只占水柱中蓝藻增长量的 $2.15 \%^{[14]}$. 由于底泥释放量对于水柱 中蓝藻生物量的贡献较小, 所以有必要对水柱中蓝藻生长进行测定并与底泥释放量比较, 确定两种水华蓝藻来源 对于蓝藻总生物量的相对贡献. 此外, 浅水湖泊中蓝藻复苏的驱动因子、底泥中蓝藻的复苏释放量、水柱中蓝藻的 生长、释放量与水柱中生长量的比较等问题,都没有得到定量的研究和深人的探索. 本文在去年蓝藻复苏研究的 基础上,自行设计了藻类原位培养器并对蓝藻的原位生长率进行测定;同时利用已有的蓝藻复苏收集器收集从底 泥向水柱迁移的蓝藻, 最后对两种途径进行比较以确定它们对于蓝藻生物量的相对贡献. 该研究旨在为蓝藻复苏 过程、生物量的累积和水华的形成提供实验数据和理论根据.

\section{1 材料与方法}

\section{1 采样点选取与采样}

太湖是我国第三大淡水湖泊 $\left(30^{\circ} 56^{\prime}-31^{\circ} 34^{\prime} \mathrm{N} ; 119^{\circ} 54^{\prime}-120^{\circ} 36^{\prime} \mathrm{E}\right.$ ), 面积为 $2338 \mathrm{~km}^{2}$, 最大水深 $2.6 \mathrm{~m}$, 平均水 深 $1.9 \mathrm{~m}$,多年平均水交换天数 $264 \mathrm{~d}$. 梅梁湾是太湖中富营养区域,该区域每年都会出现严重的水华. 采样点位于 梅梁湾内 (图 1).

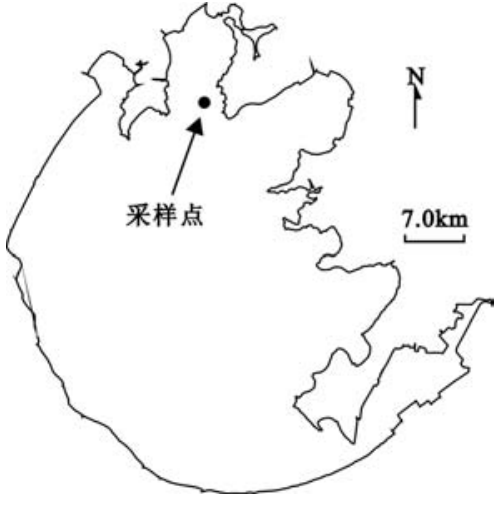

图 1 太湖蓝藻复苏研究的采样点

Fig. 1 Study site of cyanobacteria recruitment in Lake Taihu

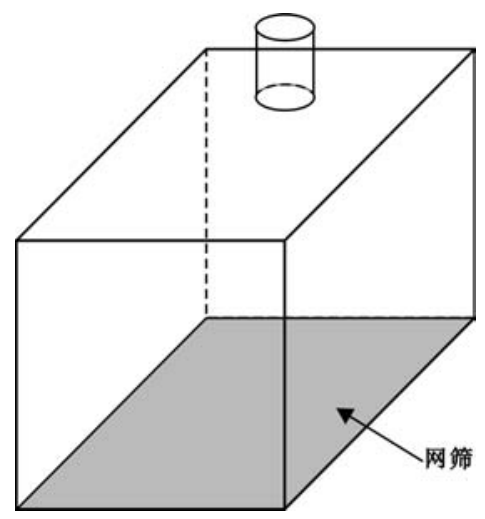

图 2 藻类原位生长培养器

Fig. 2 In situ phytoplankton chamber for growth measurement

\section{2 样品采集和分析}

根据 Cao 等人 ${ }^{[14]}$ 设计的藻类复苏收集器 $(20 \mathrm{~L})$ 来监测太湖中从底泥 复苏的蓝藻. 采样捕捉器具体的特征和用途参照曹焕生等的设计. 采样时 把收集器安放在底泥表面, 通过 $12 \mathrm{~V}$ 电动泵将捕捉器内的水抽出, 外围的 水通过 $3 \mu \mathrm{m}$ 的网篮进人捕捉器, 从捕捉器里连续抽出 $25 \mathrm{~L}$ 水样. 这样能 够去除收集器中原有的蓝藻. 复苏采集器在底泥表明放置 $3 \mathrm{~d}$, 让底泥中的 蓝藻上移进人捕捉器, 再通过电洜抽取 $2.5 \mathrm{~L}$ 水样. 其中的 $1 \mathrm{~L}$ 及时用 Lugolś 液固定用于鉴定蓝藻的组成; 剩余水样带回实验室用 $\mathrm{GF} / \mathrm{C}$ 滤膜 (Whatman, 英国) 过滤及时冷冻保存, 以用来测量藻蓝素含量. 我们用藻 蓝素表征蓝藻的生物量 ${ }^{[15]}$, 微囊藻的生物量根据其在整个蓝藻群落中的 比例确定.

浮游生长的蓝藻在透明有机玻璃培养器培养后测定其生物量 (图 2). 培养室容积 $50 \mathrm{~L}$ 底部覆盖 $3 \mu \mathrm{m}$ 的网篮 (Sefar 瑞士) 能够阻挡群体微 囊藻乃至单个细胞的进出. 实验时将其固定在采样点的钢架上. 湖水的加 人以及培养后从培养室的取出是通过培养器上部的塞子进行的, 该塞子 在培养期间用同样材料的有机玻璃封住. 为了监测浮游动物捕食对微囊 藻生长速率的影响, 湖水通过 $63 \mu \mathrm{m}$ 网篮进人培养器. 培养器放置在 0.5 $\mathrm{m}$ 深处 $3 \mathrm{~d}$. 通过测定培养前后对培养器中的色素的含量变化来计算比生 长率和生长量.

水样在通过 $\mathrm{GF} / \mathrm{C}$ 滤膜过滤后冻融一次,然后研磨滤膜,用 $90 \%$ 丙酮 抽提后定容后用苂光分光光度计检测 $(R F-5301 \mathrm{PC} \text { Shimadzu, 日本 })^{[15]}$.

\section{2 结果与讨论}

\section{1 底泥中蓝藻的复苏释放}

太湖中蓝藻的复苏和生长在 3 月份已经开始. 从所得数据来看 (图 3 ), 就整个蓝藻的复苏期而言, 蓝藻的复苏从 3 底到 6 底呈增长趋势, 在 4 月份达到最大复苏率, 之后略有降低. 同时期水柱中蓝藻的生长也呈现类 似的增长趋势, 也是在 4 月份达到最高的比生长率. 但是 5 月初有显著的 降低, 从 5 月底开始回升直到 6 月份. 此时太湖局部有微囊藻水华的出 
现. 2004 年太湖中蓝藻的复苏也是从 3 月份开始, 之后的几个月呈现上升的趋势, 在 4 月份有显著的增加 ${ }^{[14]}$. 迄今 为止, 蓝藻的复苏在深水湖泊中的研究较多, 相对而言, 浅水湖泊中蓝藻复苏的没有得到深人的研究. 复苏为水柱 中蓝藻的生长提供种源. 多数湖泊种蓝藻的复苏均表明底泥提供的蓝藻致占水体中最大生物量的 $0.05 \%-$ $5 \%{ }^{[11]}$. 太湖中蓝藻的复苏只占水柱中蓝藻生物量的 $2.15 \%{ }^{[14]}$. 即使如此, 模型研究表明如果忽略蓝藻的复苏, 夏 季的水华会减少 $50 \%$ 的生物量 ${ }^{[13]}$. 陶益等通过室内模拟研究了蓝藻在不同温度下的复苏, 结果表明蓝藻在 $14^{\circ} \mathrm{C}$ 时才开始复苏, 在 $18-20^{\circ} \mathrm{C}$ 达到复苏的最大量 ${ }^{[16]}$. 李阔宇等也发现铜绿微囊藻在 $15^{\circ} \mathrm{C}$ 开始从底泥释放到水柱中, $20^{\circ} \mathrm{C}$ 时蓝藻大量复苏 ${ }^{[17]}$. 这时由于蓝藻的复苏与其浮力的恢复有密切的关系, 只有在其密度小于湖水密度时才 会上浮; 而蓝藻在 $12^{\circ} \mathrm{C}$ 才会慢慢恢复浮力. 本文的蓝藻方式开始湖水温度为 $11.5^{\circ} \mathrm{C}$, 此时蓝藻的复苏已经开始.

\section{2 水柱中蓝藻的生长}

水柱中蓝藻的生长与种群的扩大, 优势的确立并最 终与水华的形成具有密切的关系. 太湖中蓝藻的比增长 率在复苏期呈现波动性 (图 4), 从三月到四月比增长率 降低,五月初达到最大值, 之后又降低. 总体而言, 浮游 动物对蓝藻比生长率没有明显的影响; 只有在五月初才 表现出显著的捕食作用从而抑制蓝藻的生长 $(P<0.01$, $n=3)$. 其他的研究结果也显示浮游动物对于蓝藻的生 长没有固定的作用模式 ${ }^{[18]}$. Davis and Walsby 在 Blelham Tarn 也观察到 Planktothrix rubescens 在 $25^{\circ} \mathrm{C}$ 的比生长率 为 $0.33 \mathrm{~d}^{-1[19]}$. 原位藻类的生长率的研究较少. 已有文 献表明, 在实验条件下指数生长期的铜绿微囊藻具有明 显的细胞分裂周期,但是在静止期和成熟期则没有; 湖 泊中同一区域铜绿微囊藻的原位生长昼夜呈现波动性 变化, 但是昼夜前后的增值率大致相等; 此外, 研究还表 明空间位置的变化对铜绿微囊藻的生长具有明显的影 响, 近岸区域比开阔区域的生长率高 ${ }^{[20]}$. 另外, 环境因子 对于蓝藻的增长率也有直接的作用. 在波动性的光照 下, 铜绿微囊藻的原位生长率会受到抑制 ${ }^{[21]}$.

\section{3 蓝藻底泥复苏释放和水柱中生长量比较}

底泥中的蓝藻通过两种途径影响水柱中蓝藻的生 物量. 一是在水柱中的蓝藻恢复活性以前, 底泥蓝藻恢 复代谢、释放到水柱中并增殖. 此外, 底泥中的蓝藻也增 殖并向水柱释放直接增加其生物量. 因此水柱中的蓝藻 生物量有两种来源: 底泥的释放和水柱蓝藻的增殖. 两 者的相对贡献目前还未有报道. 文中对太湖中蓝藻的复 苏释放和水柱增殖做了比较 (表 1). 复苏释放相对水柱 的生长量而言, 底泥的释放只占很小的一部分, 最多为 水柱增长量的 $2.3 \%$. 以前的结果 $(2.15 \%)$ 是整个复苏 过程前后水柱中蓝藻生物量的差值结算的出的 ${ }^{[14]}$. 本次 实验同步比较了蓝藻复苏量和水柱生长量的实时结果, 并未考虑蓝藻复苏对于浮游蓝藻生物量的种源注人. 此 外, 统计结果显示浮游动物对于两者的相对贡献无明显 的影响 $(P>0.05)$. 因此, 对于水柱中蓝藻生物量的增加

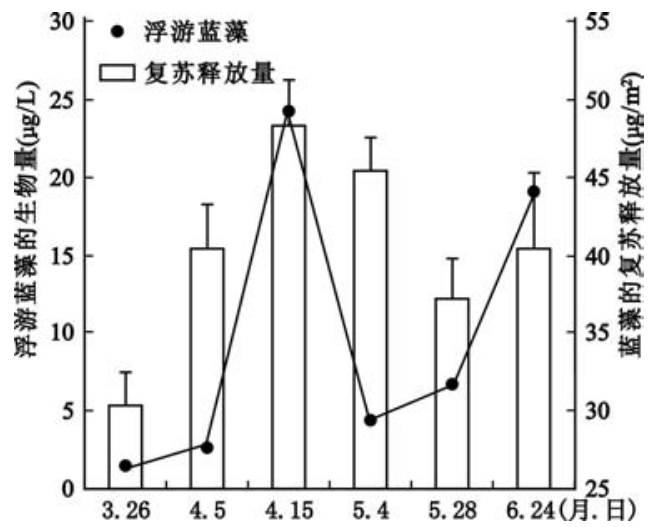

图 3 太湖中底泥表层蓝藻复苏释放量和 水柱中蓝藻生物量的变化 (2005)

Fig. 3 Recruitment of cyanobacteria from surface sediments and their pelagic abundance in Lake Taihu

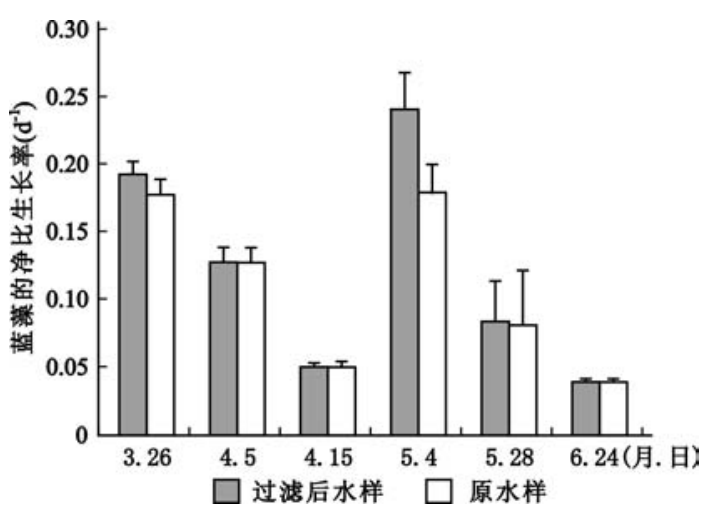

图 4 太湖水柱中蓝藻的原位生长率

Fig. 4 Net growth rate of column cyanobacteria with and without zooplankton in Lake Taihu 和优势的确立,则需更多关注水柱中的蓝藻. 
表 1 太湖蓝藻底泥复苏释放量和水柱中生长量的比较

Tab. 1 Comparison of cyanobacteria recruitment with its pelagic growth in Lake Taihu

\begin{tabular}{ccccccc}
\hline & 3.26 & 4.5 & 4.15 & 5.4 & 5.28 & 6.24 \\
\hline 释放量 $\left(\mu \mathrm{g} / \mathrm{m}^{2}\right)$ & 30.4 & 40.3 & 48.2 & 45.4 & 37.1 & 40.3 \\
过滤后水样生长量 $(\mathrm{mg} /$ column $)$ & 1.3 & 1.9 & 6.0 & 4.7 & 2.8 & 3.5 \\
原水样生长量 $(\mathrm{mg} / \mathrm{column})$ & 1.5 & 2.2 & 6.6 & 5.5 & 3.0 & 4.0 \\
释放量 v. s. OW 生长量 $(\%)$ & 2.3 & 2.1 & 0.8 & 1.0 & 1.3 & 1.1 \\
释放量 v. s. ZR 生长量 $(\%)$ & 2.0 & 1.8 & 0.7 & 0.8 & 1.2 & 1.0 \\
\hline
\end{tabular}

\section{3 结论}

本文通过对太湖蓝藻复苏进行原位的实验研究, 得出如下的结论:

(1) 太湖底泥中蓝藻的复苏从三月到六月呈现波动性的增长, 并在 4 月份达到最大量, 这与水柱中蓝藻生物 量的变化趋势一致.

(2) 太湖水柱中蓝藻的增殖呈现波动性的变化,在五月初达到最大值; 浮游动物对于蓝藻的比生长率没有显 著的影响.

(3) 底泥中复苏释放的蓝藻只占水柱中蓝藻生长量的 $2.0 \%$ 左右, 更多的研究要关注水柱中蓝藻生长和优势 的确立.

\section{4 参考文献}

[1] Oliver R L, Ganf G G. Freshwater blooms. In: Whitton BA, Potts M, eds. The ecology of cyanobacteria-their diversity in time and space, Dordrecht: Kluwer Academic Publisher, 2000: 149 - 194.

[2] Paerl H W. Nuisance phytoplankton blooms in coastal, estuaries, and inland waters. Limnol \& Oceanogr, 1988, 33: $823-847$.

[3] Trimbee A M, Harris G P. Phytoplankton population dynamics of a small reservoir: use of sedimentation traps to quantify the loss of diatoms and recruitment of summer bloom-forming blue-green algae. J Plankton Res, 1984, 6: $897-918$.

[4] Takamura N, Yasuno M. Sedimentation of phytoplankton populations dominated by Microcystis in a shallow lake. J Plankton Res, 1988, 10: 283 - 299.

[5] Visser P M, Ibelings B W, Mur L R. Autumnal sedimentation of Microcystis spp. as a result of an increase in carbonate ballast at reduced temperature. J Plankton Res, 1995, 17: 919 - 933.

[6] Preston T, Stewart W D P, Reynolds C S. Bloom-forming cyanobacterium Microcystis aeruginosa overwinters on sediment surface. Nature, 1980, 288: 365 - 367.

[7] Fallon R D, Brock T D. Overwintering of Microcystis in Lake Mendota. Freshwat Biol, 1981, 11: 217 - 226.

[8] Barbiero R P, Kann J. The importance of benthic recruitment to the population development of Aphanizomenon flos-aquae and internal loading in a shallow lake. J Plankton Res, 1994, 16: 1581 - 1588.

[9] Hansson L A, Rudstam L G, Johnson T B. et al. Patterns in algal recruitment from sediment to water in a dimictic, eutrophic lake. Can J Fish Aqua Sci, 1994, 51: 2825 - 2833.

[10] Head R M, Jones R I, Bailey-Watts A E. An assessment of the influence of recruitment from the sediment on the development of planktonic populations of cyanobacteria in a temperate mesotrophic lake. Freshwat Biol, 1999, 41 : $759-769$.

[11] Brunberg A-K, Blomqvist P. Recruitment of Microcystis from lake sediments : the importance of littoral inocula. $J$ Phycol, 2003, 39: $58-63$.

[12] 孔繁翔, 高 光. 大型浅水富营养化湖泊中蓝藻水华形成机理的思考. 生态学报, 2005,25(3): $589-595$ 
[13] Verspagen J M H, Snelder E O F M, Visser P M, et al. Benthic-pelagic coupling in the population dynamics of the harmful cyanobacterium Microcystis. Freshwat Biol, 2005, 50: $854-867$.

[14] Cao H S, Kong F X, Tan J K, et al. Recruitment of total phytoplankton, chlorophytes and cyanobacteria from lake sediments recorded by photosynthetic pigments in a large, shallow lake (Lake Taihu, China). Internat Rev Hydrobiol, 2005.

[15] 阎 荣, 孔繁翔, 韩小波. 太湖底泥表层越冬藻类群落动态的苂光分析法初步研究. 湖泊科学, 2004,16 (2) : $163-167$.

[16] 陶 益, 孔繁翔, 曹焕生, 张晓峰. 太湖底泥水华蓝藻复苏的模拟. 湖泊科学, 2005, 17(3): 231 - 236 .

[17] 李阔宇, 宋立荣, 万 能. 底泥中铜绿微囊藻复苏和生长特性的研究. 水生生物学报, 2004, 28 (2): 113 -118 .

[18] Huovinen P S, Brett M T, Goldman C R. Temporal and vertical dynamics of phytoplankton net growth in Castle Lake, California. J Plankton Res, 1999, 21 : 373 - 385.

[19] Davis P A, Walsby A E. Comparison of measured growth rates with those calculated from rates of photosynthesis in Planktothrix spp. isolated from Blelham Tarn, English Lake District. New Phytol, 2002, 156: 225 - 239

[20] Tsujimura S. Application of the frequency of dividing cells techniques to estimate the in situ growth rate of Microcystis (cyanobacteria). Freshwat Biol, 2003, 48:2009 - 2024.

[21] Mitrovic S M, Howden C G, Bowling L C, et al. Unusual allometry between in situ growth of freshwater phytoplankton under static and fluctuating light environments: possible implication for dominance. J Plankton Res, $2003,25: 517-526$. 